\title{
Combination of novel and tradition biomarkers to enhance diagnostic sensitivity and specificity for early diagnosis of rheumatoid arthritis and seronegative rheumatoid arthritis
}

Accepted: 26/8/2018

\begin{abstract}
Background and objective: Rheumatoid arthritis is an autoimmune disease. This study investigated the potential value of combining cartilage oligometric matrix protein, anti-cyclic citrullinated protein, and 14-3-3 eta protein with traditional biomarkers to reduce the diagnostic gap.
\end{abstract}

Methods: This case-control study included 46 male and female patients and 42 age- and gender-matched adults as control group. The biomarkers were measured using ELISA technique.

Results: Tests for anti-cyclic citrullinated protein and cartilage oligometric matrix protein are excellent tools to diagnose rheumatoid arthritis because anti-cyclic citrullinated protein and cartilage oligometric matrix protein are associated with the highest ROC area. The validity of the test for 14-3-3 eta protein, which is a good test to predict rheumatoid arthritis, ranks second. The optimum cut-off values for high cartilage oligometric matrix protein, high anti-cyclic citrullinated protein, and high $14-3-3$ eta protein were $\geq 0.242 \mu \mathrm{g} / \mathrm{L}$, $\geq 0.566 \mathrm{ng} / \mathrm{L}$, and $\geq 0.145 \mathrm{ng} / \mathrm{L}$, respectively. $14-3-3 \eta$ protein, cartilage oligometric matrix protein status as a parallel combination which is considered as a wonderful combination in classification criteria for rheumatoid arthritis. Parallel combination, both criteria two tests are positive, namely "high cartilage oligometric matrix protein $(\geq 0.242)+$ high $14-3-3 \eta$ protein $(\geq 0.145$ )" was associated with a perfect test, that the patients have rheumatoid arthritis (sensitivity $100 \%$, specificity $100 \%$, accuracy $100 \%$, positive predictive value at pre-test probability $50 \%$ and $90 \%=100 \%$ ). A positive test using this combination is $100 \%$ diagnostic and establishes a possible diagnosis of rheumatoid arthritis with $100 \%$, while a negative test would exclude a possible diagnosis of rheumatoid arthritis with $100 \%$ confidence.

Conclusion: Results confirmed that high serum level of cartilage oligometric matrix protein, anti-cyclic citrullinated protein, and 14-3-3 eta protein are significantly associated with increased risk for rheumatoid arthritis, demonstrating the potential value of combining these new biomarkers with traditional biomarkers to enhance diagnostic sensitivity and specificity and ultimately reduce the diagnostic gap.

Keywords: Rheumatoid arthritis; Cartilage oligometric matrix protein; Anti-cyclic citrullinated protein; 14-3-3 eta protein.

\section{Introduction}

Rheumatoid arthritis (RA) is a chronic, autoimmune disease characterized by synovial inflammation and joint degradation. The economic burden explains the increasing amount of attention that has been devoted to arthritis and in finding pharmacological agents to control this disease. However, RA is difficult to diagnose. To facilitate diagnosis during the early stages of RA, when often not all clinical symptoms are manifest among classical serological markers RF, ESR, $\mathrm{CRP}$, and anti-cyclic citrullinated protein (ACCPA). An increasing amount of attention has been recently devoted to the

${ }^{1}$ Department of Clinical Analysis, College of Pharmacy, Hawler Medical University, Erbil, Iraq.

* Correspondence: shatha.mustafa@hmu.edu.krd 
potential role of these biomarkers in the early diagnosis of RA and seronegative RA. Studies have identified many novel candidate biomarkers for RA, and the sensitivity of these markers is considerably low in the early disease phase. Recent findings have shown that ACCPA is expressed several years prior to the onset of clinical symptoms of RA. Thus, the initial events that induce ACCPA generation apparently take years to develop into a full-blown disease. ${ }^{1}$ ACCPA plays a key role in the pathophysiology of RA; moreover, ACCPA is specific for RA and detected early in the disease course and thus can possibly predict disease development. $^{2}$ Combinations of several serologic markers have been used to assist in RA diagnosis. Moreover, ACCPA was included in the 2010 RA classification criteria. $^{3}$ Prevention of RA in high-risk subjects requires early detection of RA to determine the effectiveness and cost of preventive measures. ${ }^{4}$ Despite the strong diagnostic value of traditional biomarkers, novel serological biomarkers are strongly needed to further improve the early diagnosis of RA and seronegative RA, to improve the diagnostic sensitivity and specificity to reduce the diagnostic gap. This study investigated the predictors COMP, ACCPA, and 14-3-3 eta protein, which can all be detected early in the course of RA. Any biomarker that can contribute to early diagnosis and clearly indicates the stratification of the persistence and severity of RA is of most interest in RA patients. This study compared the accuracy of the different combinations of COMP, ACCPA, and14-33 eta protein, which are potential biologic markers for arthritis and which have shown a promising application in early diagnosis of RA and seronegative RA. Moreover, this study compared the present results with previous findings to design studies that will elucidate the mechanism of action of these biomarkers at the population level and ultimately provide insight into the use of these biomarkers as a molecular target in
RA therapy. This study provides further evidence for the re-evaluation of the American College of Rheumatology 2012 (ACR 2012) classification criteria for $R A{ }^{5}$ as well as includes 14-3-3 eta protein and COMP status as criteria for RA classification. Tests for ACCP, COMP, and 14-3-3n protein are apparently simple, non-invasive, and independent parameters for diagnosis and assessment of disease severity and are useful in early diagnosis of RA. These focused parameters have emerged as candidate biomarkers for early detection of RA and seronegative RA. This study was designed to test the hypothesis that serum levels of the focused parameters are altered and related to the development of RA. Therefore, this study was performed to elucidate whether the combination of the focused parameters and traditional biomarkers, namely, RF, $E S R$, and CRP, reduces the diagnostic gap and improves diagnostic sensitivity and specificity. These selected biomarkers may, therefore, improve the early diagnosis of RA. These biomarkers are tools that can possibly aid in diagnosis and subsequent risk stratification and secondary prevention. Moreover, these biomarkers may serve as a guide in the selection of therapy and serve as a target for therapy. Consequently, efforts have been devoted in the identification of risk factors for RA, and this approach may allow early diagnosis or identification of people who are likely to develop RA; consequently, prevention and treatment may be initiated earlier. This study aimed to investigate the potential value of combining COMP, ACCPA, and 14-3-3n with traditional biomarkers, namely, RF, ESR, and CRP, in reducing the diagnostic gap, in improving diagnostic sensitivity and specificity, and in assessing the effect of other confounding factors, such as age, gender, and BMI.

\section{Methods}

This case-control study was performed at the College of Pharmacy, Hawler Medical 
University between October 2015 and December 2016 on 46 male and female patients, who were conveniently selected and diagnosed by a specialist through clinical examination. The diagnosis was confirmed by laboratory tests at the Rheumatic Center after exclusion of other diseases based on clinical history, laboratory investigations, and clinical examination. For comparison, 42 apparently healthy were enrolled as a control group; these individuals were also conveniently selected and have answered the baseline questionnaire on several risk factors. The control group was confirmed to be in normal condition through clinical, biochemical examination like fasting blood sugar, lipid profile (cholesterol, triglyceride, VLDL, LDL, HDL), electrolytes estimation, calcium, liver function test (GOT, GPT, gamma glutamyltransferase, alkaline phosphatase, bilirubin), kidney function (urea, creatinine, total protein, albumin), uric acid, and hematological examinations (complete blood picture, RBC, WBC, platelet, ESR, hemoglobin, direct Coombs test. Allpatients fulfilled the American College of Rheumatology 2012(ACR 2012) criteria for RA. ${ }^{5}$ Patients with RA were diagnosed by a specialist physician (Rheumatologist) after clinical examination and confirmed by laboratory tests at Rheumatic Center in Erbil Teaching Hospital. During the period of study, no antioxidants as dietary supplements such as vitamins or minerals were given to the patients and control subjects. All procedures were performed in accordance with established ethical standards. Biochemical, hematological, and all clinicopathological data of the patients were obtained from their medical records. Verbal informed consents were obtained from all participants prior to their enrolment in this study. The protocol used in this research was approved by the Ethics Committee for Medical Research of the College of Pharmacy, Hawler Medical University.

Sample Collection: Blood samples (10 ml) for fasting blood test were extracted from the participants, coagulated for $30 \mathrm{~min}$, and then centrifuged for $15 \mathrm{~min}$ at 2500-3500 rpm. Sera were separated and divided into several portions and then placed into plastic plain tubes for biochemical tests. The sera were stored at $-80{ }^{\circ} \mathrm{C}$ at the Medical Research Center, Hawler Medical University until the day of the analysis within 2-3 months. The sera samples were prepared for measurement by warming (thawing) the frozen sera at room temperature. Both groups were completed the baseline questionnaire concerning several risk factors. The focused parameters were measured using ELISA technique at the Medical Research Center, Hawler Medical University.

Statistical Analysis: Data were analyzed using the statistical package for the social sciences (version 18). The numerical variables were presented in the form of mean and SD, SE, median, range, percentiles, probability, confidence interval, the statistical tests that were used chi-square, student t-test, Mann- Whitney, the Kruskal-Wallis test was used to test the statistical significance of difference in median of a non-normally distributed variable between more than two groups, frequency distribution, optimum cut-off value. The determination of the cut-off value mostly depends on receiver operating characteristic (ROC) analysis where the sensitivity and specificity of the test are nearly equal as well as for determination of the cut-off value. We need to know the pretest probability of the disease. The ROC (receiver operating characteristic curve) analysis was used to calculate the area under the curve for each tested parameter in the context of differentiation between two groups. The ROC area ranges between 0.5 (equivocal or totally useless parameter) to 1 , which is associated with a perfect test. The area under the curve gives an idea about the usefulness of the test and helps in comparing it to the other tests. The closer the area to one (ideal test), the more valid 
it is. An odds ratio is a measure of association between the presence or absence of two properties, and the associated confidence interval indicates the degree of uncertainty associated with that ratio. Pre-test probability, which is known as a differential diagnosis that the physician should have taken from the signs and symptoms of each patient (guess). $P$ value of $<0.05$ was considered significant.

\section{Results}

\section{Characteristics of the Studied Groups:}

The studied subjects were 21-69 years old.
No significant difference in age and BMI was observed between patients (44.8 years old and $27.6 \mathrm{~kg} / \mathrm{m}^{2}$, respectively) and controls (45.4 years old and $28 \mathrm{~kg} / \mathrm{m}^{2}$, respectively). More female than male subjects were included in both the patient and control groups $(80.4 \%$ and $85.7 \%$, respectively) (Table 1 ). The mean blood ESR was significantly higher (44.1 $\mathrm{mm} / \mathrm{h})$ in patients than in controls $(22.2$ $\mathrm{mm} / \mathrm{h}$ ) (Table 1).

Table 1: Characteristic of studied groups, case-control difference in mean age, BMI and gender distribution.

\begin{tabular}{|c|c|c|c|}
\hline & \multicolumn{2}{|c|}{ Studied group } & \multirow[b]{2}{*}{$P$ value } \\
\hline & Healthy controls & Cases (RA) & \\
\hline Age (years) & & & $0.80[N S]$ \\
\hline Range & (21 to 69$)$ & (21 to 69$)$ & \\
\hline Mean & 45.4 & 44.8 & \\
\hline SD & 12.1 & 12.1 & \\
\hline SE & 1.87 & 1.78 & \\
\hline $\mathbf{N}$ & 42 & 46 & \\
\hline BMI (Kg/m2) & & & $0.70[\mathrm{NS}]$ \\
\hline Range & (20 to 45.7 ) & (18.8 to 43.3 ) & \\
\hline Mean & 28 & 27.6 & \\
\hline SD & 5.3 & 5.6 & \\
\hline SE & 0.82 & 0.83 & \\
\hline $\mathbf{N}$ & 42 & 46 & \\
\hline Female & $36(85.7) \%$ & $37(80.4) \%$ & \\
\hline Male & $6(14.3) \%$ & $9(19.6) \%$ & $\begin{array}{c}P(\text { Chi-square })= \\
0.9[\mathrm{NS}]\end{array}$ \\
\hline ESR (mm/hour) & & & $<0.001$ \\
\hline Range & (13 to 38$)$ & (2 to 118$)$ & \\
\hline Mean & 22.2 & 44.1 & \\
\hline SD & 5.2 & 26.1 & \\
\hline SE & 0.81 & 3.85 & \\
\hline $\mathbf{N}$ & 42 & 46 & \\
\hline
\end{tabular}


Case-Control Difference in Selected Markers:

In Table 2, Mann Whitney test was used to compare between the mean ranks, there were significant differences between the patients and control groups in mean rank values regarding the studied parameters COMP, ACCPA, and 14-3-3 eta protein $P<0.001$.
Risk for RA Based on RF and CRP Test Results:

The risk of RA was significantly increased by 14.2 times in RF- or CRP-positive subjects (Table 3 ). A significantly higher proportion $(73.9 \%)$ of RA cases were RF- or CRP-positive compared with the positivity rate of $16.7 \%$ among controls.

Table 2: The case-control difference in mean rank of selected biochemical measurements.

\begin{tabular}{|c|c|c|c|}
\hline & \multicolumn{2}{|c|}{ Study group } & \multirow{2}{*}{$P$ value } \\
\hline & Healthy controls & Cases (RA) & \\
\hline cartilage oligomeric matrix protein $(\mu \mathrm{g} / \mathrm{L})$ & & & $<0.001$ \\
\hline Range & (0.047 to 1.11$)$ & (0.285 to 0.695$)$ & \\
\hline Median & 0.081 & 0.4075 & \\
\hline Inter-quartile range & $(0.076$ to 0.093$)$ & $(0.375$ to 0.445$)$ & \\
\hline $\mathrm{N}$ & 42 & 46 & \\
\hline Mean Rank & 24.8 & 62.5 & \\
\hline Anti-citrullinated protein antibodies (ng/L) & & & $<0.001$ \\
\hline Range & (0.099 to 1.471$)$ & (0.43 to 1.95$)$ & \\
\hline Median & 0.164 & 0.7 & \\
\hline Inter-quartile range & $(0.125$ to 0.21$)$ & $(0.65$ to 0.75$)$ & \\
\hline $\mathrm{N}$ & 42 & 46 & \\
\hline Mean Rank & 23.8 & 63.4 & \\
\hline 14-3-3 eta protein (ng/L) & & & $<0.001$ \\
\hline Range & $(0.033$ to 2.02$)$ & $(0.225$ to 0.505$)$ & \\
\hline Median & 0.0505 & 0.245 & \\
\hline Inter-quartile range & $(0.046$ to 0.058$)$ & $(0.235$ to 0.27$)$ & \\
\hline $\mathrm{N}$ & 42 & 46 & \\
\hline Mean Rank & 27 & 60.5 & \\
\hline
\end{tabular}

Table 3: The risk of having RA by RF and CRP tests.

\begin{tabular}{lccccccc}
\hline \multicolumn{7}{c}{ Studied group } \\
& Healthy controls & \multicolumn{2}{c}{ Cases (RA) } & & & \\
& $\mathbf{N}$ & $\%$ & $\mathbf{N}$ & $\%$ & OR & $\mathbf{9 5 \%}$ Cl OR & P value \\
\hline RF & & & & & & & $<0.001$ \\
Negative & 35 & 83.3 & 12 & 26.1 & Reference & & \\
Positive & 7 & 16.7 & 34 & 73.9 & 14.2 & $(5.0-40.3)$ & \\
CRP & & & & & & & $<0.001$ \\
Negative & 35 & 83.3 & 12 & 26.1 & & & \\
Positive & 7 & 16.7 & 34 & 73.9 & 14.2 & $(5.0-40.3)$ & \\
\hline
\end{tabular}


Validity of Selected Measurements:

The validity of the three selected quantitative biochemical measurements was compared with that of ESR, RF, and CRP when tested for RA diagnosis differentiating it from controls. As shown in Table 4, tests for ACCPA and COMP are excellent tools to diagnose RA because these biomarkers were associated with the highest ROC area (0.929 and 0.950, respectively). The validity of the test for 14-3-3 eta protein, which is a good tool (ROC area is 0.881 ) to predict RA, ranked second followed by the validity of the test for the three remaining markers, namely ESR, RF, and CRP. These tests showed a fair performance (ROC area is $>0.7$ ). The determination of the cut-off values depends on the ROC analysis where the sensitivity and specificity of the test are nearly equal, so the optimum cut-off value for COMP, ACCPA, $14-3-3$ eta were set at $\geq 0.242$, $\geq 0.566$, $\geq 0.145$ respectively, the optimum cut-off value for ESR was set at $\geq 27.5 \mathrm{~mm} /$ hour, the RF test is $73.9 \%$ sensitive and $83.3 \%$ specific (Table 5 ).

Table 4: ROC area for selected measurements as a test to diagnose RA differentiating them from controls.

\begin{tabular}{lcc}
\hline & ROC area & $\boldsymbol{P}$ value \\
\hline Anti-citrullinated protein antibodies & 0.950 & $<0.001$ \\
cartilage oligomeric matrix protein (COMP) & 0.929 & $<0.001$ \\
14-3-3 eta protein & 0.881 & $<0.001$ \\
ESR & 0.787 & $<0.001$ \\
RF & 0.786 & $<0.001$ \\
CRP & 0.786 & $<0.001$ \\
\hline
\end{tabular}

Table 5: Validity for selected parameters as a test to diagnose RA cases differentiating them from controls.

\begin{tabular}{|c|c|c|c|c|c|c|}
\hline \multirow[b]{2}{*}{ Positive $\geq$ cut-off } & \multirow[b]{2}{*}{ Sensitivity } & \multirow[b]{2}{*}{ Specificity } & \multirow[b]{2}{*}{ Accuracy } & \multicolumn{2}{|c|}{$\begin{array}{l}\text { PPV at pre-test } \\
\text { probability = }\end{array}$} & \multirow{2}{*}{$\begin{array}{c}\text { NPV at pre-test } \\
\text { probability = } \\
10 \%\end{array}$} \\
\hline & & & & $50 \%$ & $90 \%$ & \\
\hline \multicolumn{7}{|c|}{ Anti-citrullinated protein antibodies } \\
\hline$\geq 0.418$ (highest sensitivity) & 100.0 & 88.1 & 94.3 & 89.4 & 98.7 & 100.0 \\
\hline$\geq 0.566$ (Optimal cut-off) & 93.5 & 95.2 & 94.3 & 95.2 & 99.4 & 99.2 \\
\hline$\geq 1.711$ (highest specificity) & 2.2 & 100.0 & 48.9 & 100.0 & 100.0 & 90.2 \\
\hline \multicolumn{7}{|l|}{$\begin{array}{l}\text { Cartilage oligomeric matrix protein } \\
\text { (COMP) }\end{array}$} \\
\hline $\begin{array}{l}\geq 0.242 \text { (highest sensitivity, specificity, } \\
\text { and Optimal cut-off) }\end{array}$ & 100.0 & 92.9 & 96.6 & 93.3 & 99.2 & 100.0 \\
\hline \multicolumn{7}{|l|}{ 14-3-3 eta protein } \\
\hline $\begin{array}{l}\geq 0.145 \text { (highest sensitivity, specificity, } \\
\text { and Optimal cut-off) }\end{array}$ & 100.0 & 88.1 & 94.3 & 89.4 & 98.7 & 100.0 \\
\hline \multicolumn{7}{|l|}{ ESR } \\
\hline$\geq 14.0$ (highest sensitivity) & 93.5 & 4.8 & 51.1 & 49.5 & 89.8 & 86.8 \\
\hline$\geq 27.5$ (Optimal cut-off) & 76.1 & 85.7 & 80.7 & 84.2 & 98.0 & 97.0 \\
\hline$\geq 38.5$ (highest specificity) & 47.8 & 100.0 & 72.7 & 100.0 & 100.0 & 94.5 \\
\hline Positive RF & 73.9 & 83.3 & 78.4 & 81.6 & 97.6 & 96.6 \\
\hline Positive CRP & 73.9 & 83.3 & 78.4 & 81.6 & 97.6 & 96.6 \\
\hline
\end{tabular}

PPV: Positive predictive value NPV: Negative predictive value 
The RF test was $73.9 \%$ sensitive and 83.3\% specific. Positive testing would establish a possible diagnosis of RA with $81.6 \%$ confidence in a clinical situation with equal odds of having versus not having the disease (pretest probability $=50 \%$ ). The confidence in a positive test result increases to $97.6 \%$ (pretest probability = $90 \%$ ). A negative test would exclude a possible diagnosis of RA with $96.6 \%$ confidence in a clinical situation where RA onset shows a very low probability (pretest probability $=10 \%$ ). Similar test performance was observed for CRP. Determination of the performance of the three markers at optimum cut-off value was repeated for comparison with the performance of the three possible combinations (Table 6).

\section{Discussion}

Laboratory test results for ESR, CRP, and RF provide useful information on disease activity but are not specific to RA. This study investigated the potential value of combining COMP,ACCPA, and 14-3-3 eta protein with traditional biomarkers to reduce the diagnostic gap, to improve diagnostic sensitivity and specificity, and to assess the effect of other confounding factors, such as age, gender, BMI, and seropositive factor. RA can appear at any age but typically develops between ages 30 and 60 . The incidence of RA generally increases with increasing age, consistent with the current findings. The studied subjects were 21-69 years old. No significant difference in age and BMI was observed between the patients $(44.8$ years and $27.6 \mathrm{~kg} / \mathrm{m}^{2}$, respectively) and controls (45.4 years old and $28 \mathrm{~kg} / \mathrm{m}^{2}$, respectively) (Table 1). ESR was significantly higher in patients $(44.1 \mathrm{~mm} / \mathrm{h})$ than in controls $(22.2$ $\mathrm{mm} / \mathrm{h}$ )(Table 1$)$. In addition, more females than males were included both in the patient and control groups $(80.4 \%$ and $85.7 \%$, respectively). Complex interactions exist between female sex hormones and RA. The present study assessed whether variation in COMP levels could predict RA in its early onset stage; furthermore, COMP levels were compared with those of

Table 6: Validity for selected parameters at the optimum cut-off value and a parallel combination to diagnose RA.

\begin{tabular}{|c|c|c|c|c|c|c|}
\hline \multirow{2}{*}{ Positive $\geq$ cut-off } & \multirow{2}{*}{ Sensitivity } & \multirow{2}{*}{\multicolumn{2}{|c|}{ Specificity Accuracy }} & \multicolumn{2}{|c|}{$\begin{array}{l}\text { PPV at pre-test } \\
\text { probability }=\end{array}$} & \multirow{2}{*}{$\begin{array}{c}\text { NPV at pre-test } \\
\text { probability }= \\
10 \%\end{array}$} \\
\hline & & & & $50 \%$ & $90 \%$ & \\
\hline $\begin{array}{l}\text { Cartilage oligomeric matrix } \\
\text { protein (COMP) } \geq 0.242\end{array}$ & 100.0 & 92.9 & 96.6 & 93.3 & 99.2 & 100.0 \\
\hline $\begin{array}{l}\text { Anti-citrullinated protein } \\
\text { antibodies } \geq 0.566\end{array}$ & 93.5 & 95.2 & 94.3 & 95.2 & 99.4 & 99.2 \\
\hline $14-3-3$ eta protein $\geq 0.145$ & 100.0 & 88.1 & 94.3 & 89.4 & 98.7 & 100.0 \\
\hline \multicolumn{7}{|l|}{ Parallel combination of two tests } \\
\hline $\begin{array}{l}\text { [High COMP }(\geq 0.242)+\text { High } \\
14-3-3 \text { eta protein }(\geq 0.145)]\end{array}$ & 100.0 & 100.0 & 100.0 & 100.0 & 100.0 & 100.0 \\
\hline $\begin{array}{l}{[\text { High COMP }(\geq 0.242)} \\
+ \text { High ACAP }(\geq 0.566)]\end{array}$ & 93.5 & 100.0 & 96.6 & 100.0 & 100.0 & 99.3 \\
\hline $\begin{array}{l}\text { [High } 14-3-3 \text { eta protein } \\
(\geq 0.145)+\text { High ACAP } \\
(\geq 0.566)]\end{array}$ & 93.5 & 97.6 & 95.5 & 97.5 & 99.7 & 99.3 \\
\hline PPV: Positive predictive value & \multicolumn{3}{|c|}{ NPV: Negative predictive value } & & & \\
\hline
\end{tabular}


other classical laboratory rheumatic biomarkers in combination pattern to enhance diagnostic sensitivity and specificity and ultimately reduce the diagnostic gap. COMP was recently introduced as a new marker for diagnosis and prognosis of RA, ${ }^{6}$ as well as a marker for disease severity and treatment effect. Serum COMP levels were higher in patients with aggressive RA than in those with non-aggressive RA. ${ }^{7}$ The current result showed that the median COMP was significantly higher in patients $(0.4075)$ than in controls $(0.081)$ (Table 2), consistent with previous results. ${ }^{6,8}$ ACCPA is the most specific marker for RA with a specificity of approximately $98 \%$. Thus, ACCPA is a reliable marker that confirms $R A$ diagnosis. $^{9}$ Similarly, the median ACCPA was significantly higher in patients $(0.7)$ than in controls $(0.164)$ (Table 2$)$, consistent with previous results. ${ }^{10-12}$ The current result was concordant with ${ }^{13}$ that this parameter is widely used as a diagnostic factor for RA. The current result for ACCPA demonstrates the clinical utility of ACCPA as a diagnostic tool for RA. Moreover, this study was concordant with other previous studies ${ }^{14}$ for confirming the diagnosis. The median concentration of 14-3-3 eta protein was also significantly higher in patients $(0.245)$ than in controls $(0.0505)$ (Table 2). This result is consistent with previous findings. ${ }^{10,11}$ Moreover, $14-3-3 \eta$ is a novel biomarker that is potentially useful in diagnosis and monitoring. The presence of either this protein or its auto antibodies was observed in $90 \%$ of patients with early RA. ${ }^{15}$ Its expression independently predicts $\mathrm{RA}$ development ${ }^{12}$ and is associated with a poor disease condition, resulting in a more accurate diagnosis of RA, it complements current markers and elevated the detection rate. ${ }^{10}$ Furthermore, $14-3-3$ eta protein is a novel joint-derived pro-inflammatory mediator that is implicated in RA pathogenesis. $^{10}$ Extracellular 14-3-3 eta protein at concentrations found in RA patients activates key signaling cascades and induces factors associated with RA pathogenesis, as well as highly expressed in patients showing radiographic damage and RA progression. ${ }^{10}$ Significant increase of this parameter results from the release of 14-3-3 eta protein from synovial tissue into the synovial fluid and serum of RA patients, extracellular 14-3-3 eta protein is a potent indicator and activator of the intracellular signaling pathway that up regulates factors for inflammation and joint damage, and these factors are involved in RA pathogenesis. ${ }^{16}$ Analysis of serum 14-3 -3 eta protein in relation to joint damage and progression revealed significantly increased levels of 14-3-3 eta protein relative to the baseline in patients who show radiographic evidence of damage, as well as in patients who developed radiographic progression by the end of the follow-up period. ${ }^{16}$ By using odds ratio (OR) to estimate how many times the risk of RA increased, the risk of having RA is significantly increased by $\mathrm{OR}=14.2$ times ( $\mathrm{OR}=14.2 ; 95 \% \mathrm{Cl}=5-40.3, P<0.001$ ) among subjects with positive RF or those with positive CRP. A significantly higher proportion of RA cases had a positive RF or CRP test $(73.9 \%)$ as compared to a positivity rate of $16.7 \%$ among healthy controls (Table 3 ). The validity of selected measurements using ROC area for selected measurements when used as test to diagnose RA cases differentiating them from healthy controls (Table 4). The validity of the quantitative measurements of the three selected biochemical was compared with that of ESR, RF, and CRP when tested to diagnose RA differentiating it from healthy controls. As shown in Table 4, tests for ACCPA and COMP are excellent tools for RA diagnosis because these biomarkers were associated with the highest ROC area (0.929 and 0.950, respectively). The validity of the test for 143-3 eta protein, which is a good test (ROC area 0.881 ) to predict $R A$, ranked second followed by the validity of the tests for the three remaining biomarkers, namely ESR, $\mathrm{RF}$, and CRP. The current observations confirm the role of ACCPA in RA diagnosis 
as opposed to RF and inflammatory parameters. This finding is consistent with a previous result, ${ }^{9}$ in which ACCPA test is a very useful blood test in the diagnosis of $\mathrm{RA}$, and its scores were higher than those of routine RF test for early and specific diagnosis of RA. ACCPA is not the sole specific marker for RA. Combination with COPM can enhance diagnosis, especially in the very early stage of RA. ${ }^{6}$ The optimum cut-off value for COMP was set at $\geq 0.242$ (Table 5). The test is $100 \%$ sensitive and $92.9 \%$ specific at this cut-off value. In a clinical situation, positive testing at the optimum cut-off value (obtaining a concentration of 0.242 or higher for COMP) showed a possible diagnosis of RA with $93.3 \%$ confidence at equal odds of having versus not having the disease (pretest probability $=50 \%$ ). The confidence in a positive test increased to an early perfect value $(99.2 \%)$ in a clinical situation where the disease is highly suspected based on clinical criteria and history (pretest probability $=90 \%$ ). Negative testing is highly valuable at $100 \%$ sensitivity. Negative testing (COMP value is <0.242) would exclude a possible diagnosis of RA with $100 \%$ confidence in a clinical situation where the RA onset shows a very low probability on clinical grounds (pretest probability $=10 \%)($ Table 5$)$. The optimum cut-off value for ACCPA was set at $\geq 0.566$. The test was $93.5 \%$ sensitive and $95.2 \%$ specific at this cut-off value. Positive testing at the optimum cut-off value (obtaining a concentration of 0.566 or higher for ACCPA) would establish a possible diagnosis of RA with $95.2 \%$ confidence in a clinical situation with equal odds of having versus not having the disease (pretest probability $=50 \%$ ). The confidence in a positive test increased to an early perfect value $(99.4 \%)$ in a clinical situation where the disease is highly suspected based on clinical criteria and history (pretest probability $=90 \%$ ). Positive testing at the highest specificity $(100 \%)$ and cut-off value $(\geq 1.711)$ is $100 \%$ diagnostic and would establish a possible diagnosis of RA with $100 \%$ confidence in any clinical situation. A negative test is highly valuable at the highest sensitivity (100\%) and cut-off value $(\geq 0.418)$. Negative testing (ACCPA value is $<0.418$ ) would exclude a possible diagnosis of RA with $100 \%$ confidence in a clinical situation where RA onset shows a very low probability on clinical grounds (pretest probability $=10 \%)($ Table 5$)$. The optimum cut-off value for 14-3-3 eta protein was set at $\geq 0.145$. The test is $100 \%$ sensitive and $88.1 \%$ specific at this cut-off value. Positive testing at the optimum cut-off value (obtaining a concentration of 0.145 or higher for $14-3-3$ eta protein) would establish a possible diagnosis of RA with $86.4 \%$ confidence in a clinical situation with equal odds of having versus not having the disease (pretest probability = $50 \%$ ). The confidence in a positive test increases to an early perfect value (positive predictive value $=98.7 \%$ ) in a clinical situation where the disease is highly suspected based on clinical criteria and history (pretest probability $=90 \%$ ). A negative test is highly valuable at $100 \%$ sensitivity. Negative testing (the value for 14-3-3 eta protein is <0.145) would exclude a possible diagnosis of RA with $100 \%$ confidence in a clinical situation where RA onset shows a very low probability on clinical grounds (pretest probability $=10 \%)($ Table 5$)$. The optimum cut-off value for ESR was set at $\geq 27.5 \mathrm{~mm} /$ h. The test was $76.1 \%$ sensitive and $85.7 \%$ specific at this cut-off value. Positive testing at the optimum cut-off value (ESR is $27.5 \mathrm{~mm} / \mathrm{h}$ or higher) would establish a possible diagnosis of RA with $84.2 \%$ confidence in a clinical situation with equal odds of having versus not having the disease (pretest probability $=50 \%$ ). The confidence in a positive test increased to $98 \%$ in a clinical situation where RA is highly suspected based on clinical criteria and history (pretest probability= 90\%). Positive testing at the highest specificity $(100 \%)$ and cut-off value $(\geq 38.5)$ is $100 \%$ diagnostic and would establish a possible diagnosis of RA with $100 \%$ confidence in 
any clinical situation (Table 5). The RF test was $73.9 \%$ sensitive and $83.3 \%$ specific. Positive testing would establish a possible diagnosis of RA with $81.6 \%$ confidence in a clinical situation with equal odds of having versus not having the disease (pretest probability $=50 \%$ ). The confidence in a positive test increased to $97.6 \%$ in a clinical situation where RA is highly suspected based on clinical criteria and history (pretest probability $=90 \%$ ). A negative test would exclude a possible diagnosis of RA with $96.6 \%$ confidence in a clinical situation where RA onset shows a very low probability on clinical grounds (pretest probability= 10\%) (Table 5). Similar test performance was observed for CRP. Considering the three biochemical measurements associated with the highest validity at the optimum cut-off value provided a test for RA with an accuracy of lower than $100 \%$ and a specificity of less than perfect $(<100 \%)$, one can combine the tests results to improve the validity and performance of diagnosis. Considering that two of the three previously mentioned tests (COMP $\geq 0.242$ and $14-3-3$ eta protein $\geq 0.145$ ) are already $100 \%$ sensitive, one can use a parallel combination of two tests to increase specificity. In a parallel combination of two tests or criteria, the tested subject is considered positive only if both criteria were positive, whereas the subjects are considered negative if any or both of the combined criteria were negative. As shown in Table 6, the diagnostic performance of the three markers at the optimum cut-off value was repeated to allow comparison with the performance of the three possible combinations. Only one combination (parallel combination, both criteria two tests are positive), namely "High COMP $(\geq 0.242)$ + High 14-3-3n protein ( $\geq 0.145$ )" was associated with a perfect test, it is an excellent test, it has no any mistake that the patients have RA (sensitivity 100\%, specificity $100 \%$, accuracy $100 \%$, positive predictive value at pre-test probability $50 \%$ and $90 \%=100 \%$ ), which is significantly more valid than each of the three tested criteria independently. A positive test using this combination is $100 \%$ diagnostic and establish a possible diagnosis of RA with $100 \%$, while a negative test would exclude a possible diagnosis of RA with $100 \%$ confidence also under any clinical situation and pre-test probability. Physicians should have pre-test probability, which is known as a differential diagnosis that has taken from the signs and symptoms of each patient. Combining 14-3-3n with ACCPA resulted in an identification rate of $72 \%$ compared with the $59 \%$ identification rate for ACCPA alone. ${ }^{15}$ Combining RF with ACCPA increased the diagnostic capture from $59 \%$ to $72 \%$, which further increased to $78 \%$ when $14-3-3 n$ was included. ${ }^{15}$ Furthermore, combining 14-3-3n with RF and ACCPA increased the detection rate in individuals with RA and may assist primary care physicians in triaging referrals to rheumatologists. ${ }^{17}$ This study offers a clinical significance because it identifies a new approach for early diagnosis of RA and seronegative RA to reduce medical costs, which is a massive economic burden to RA patients. ${ }^{18}$ The current results for COMP, ACCPA, and 14-3-3 eta protein confirmed the clinical utility of focused parameters as a tool in early diagnosis of RA, particularly in patients with seronegative $R A$, to reduce diagnostic gap and to enhance diagnostic sensitivity and specificityin parallel combination pattern and to reduce the medical cost among people with RA which consists a high burden to the patients with RA. Treatment during early stage possibly exerts a greater effect than treatment at a later stage in terms of halting disease progression and achieving remission. ${ }^{19}$ Recent data suggest that early therapy and efforts to achieve low-disease activity improve long-term outcomes in inflammatory arthritis. ${ }^{20}$ This study provides further evidence for the re-evaluation of the American College of Rheumatology 2012 (ACR 2012) classification criteria for $\mathrm{RA}^{14}$ and support for including $14-3-3 \eta$ protein, COMP status 
as a parallel combination which is considered as a wonderful combination in classification criteria for RA. Parallel combination, both criteria two tests are positive, namely "High COMP $(\geq 0.242)+$ High $14-3-3 \eta$ protein $(\geq 0.145)$ " was associated with a perfect test, it is an excellent test, it has no any mistake that the patients have RA (sensitivity 100\%, specificity $100 \%$, accuracy $100 \%$, positive predictive value at pre-test probability $50 \%$ and $90 \%=100 \%$ ). A positive test using this combination is $100 \%$ diagnostic and establishes a possible diagnosis of RA with $100 \%$, while a negative test would exclude a possible diagnosis of RA with $100 \%$ confidence.

\section{Conclusion}

COMP, ACCPA, and 14-3-3 eta protein may be used along with traditional markers in early diagnosis of RA, demonstrating the potential value of combining these new biomarkers with traditional biomarkers to enhance diagnostic sensitivity and specificity and to reduce the diagnostic gap.

\section{Competing interests}

The authors declare that they have no competing interests.

\section{References}

1. Schellekens GA, de Jong BA, Van den Hoogen $\mathrm{FH}$, Van de Putte LB, Van Venrooij WJ. Citrulline is an essential constituent of antigenic determinants recognized by rheumatoid arthritis-specific autoantibodies. J Clin Invest 1998; 101:273-81.

2. VanVenrooij W, Van Beers J, Pruijn G. Anti-CCP antibodies: the past, the present and the future. Nat Rev Rheumatol 2011; 7:391-8.

3. Aletaha D, Neogi T, Silman AJ, Funovits J, Felson DT, Bingham CO 3rd, et al. 2010 Rheumatoid arthritis classification criteria: an American College of Rheumatology/European League Against Rheumatism collaborative initiative. Arthritis Rheum 2010; 62(9):2569-81.

4. Bykerk VP. Strategies to prevent rheumatoid arthritis in high-riskpatients. Curr Opin Rheumatol 2011; 23(2):179-84.

5. Hochberg MC, Altman RD, April KT, Benkhalti M, Guyatt G, McGowan J, et al. Recommendations for the use of nonpharmacologic and pharmacologic therapies in osteoarthritis of the hand, hip, and knee. Arthritis Care Res (Hoboken) 2102; 64(4):465-74.

6. Aref MI, Ahmed H. Cartilage Oligomeric Matrix Protein as New Marker in Diagnosis of Rheumatoid Arthritis. Mod Chemappl 2015; 3:151.

7. Tseng S, Reddi AH, Di Cesare PE. Cartilage Oligomeric Matrix Protein (COMP): A Biomarker of Arthritis. Biomark Insights 2009; 4:33-44.

8. Andrade FD, Bender AL, da Silveira IG, Stein H, Von Mühlen CA, Staub HL. Cartilage oligomeric matrix protein/thrombospondin-5 (COMP/TSP-5) levels do not correlate to functional class in patients with rheumatoid arthritis. Clin Rheumatol 2009; 28(12):1441-2.

9. Nishimura K, Sugiyama D, Kogata Y, Tsuji G, Nakazawa T, Kawano S, et al. Meta-analysis: Diagnostic accuracy of anti-cyclic citrullinated peptide antibody and rheumatoid factor for rheumatoid arthritis. Ann Intern Med 2007; 146(11):797-808.

10. Maksymowych WP, Naides SJ, Bykerk V, Siminovitch $K A$, van Schaardenburg $D$, Boers $M$ et al. Serum $14-3-3 \eta$ is a novel marker that complements current serological measurements to enhance detection of patients with rheumatoid arthritis. J Rheumatol 2014; 41(11):2104-13.

11. Maksymowych WP, Boire G, van Schaardenburg $D$, Wichuk $S$, Turk $S$, Boers $M$, et al. 14-3-3n Autoantibodies: Diagnostic use in early rheumatoid arthritis. J Rheumatol 2015; 42(9):1587-94.

12. VanSchaardenburg $D$, Maksymowych WP, Boers M, van Beers-Tas MH, Marotta A. Serum 14-3-3 eta predicts the risk of RA development and its higher titers are associated with higher risk. Ann Rheum Dis 2014; 73:602.

13. Bizzaro N, Bartoloni E, Morozzi G, Manganelli S, Riccieri V, Sabatini $P$, et al. Anti-cyclic citrullinated peptide antibody titer predicts time to rheumatoid arthritis onset in patients with undifferentiated arthritis: results from a 2-year prospective study. Arthritis Res Ther 2013; 15(1):16.

14. Bartoloni E, Alunno A, Bistoni O, Bizzaro N, Migliorini P, Morozzi G, et al. Diagnostic value of anti-mutated citrullinatedvimentin in comparison to anti-cyclic citrullinated peptide and anti-viral citrullinated peptide 2 antibodies in rheumatoid arthritis: An Italian multicentric study and review of the literature. Autoimmun Rev 2012; 11:81520.

15. Maksymowych WP, Marotta A. 14-3-3n: a novel biomarker platform for rheumatoid arthritis. Clin Exp Rheumatol 2014; 32(5Suppl 85):35-9.

16. Maksymowych WP, van der Heijde $D$, Allaart $C F$, Landewé R, Boire G, Tak PP, et al. 14-3-3n is a novel mediator associated with the pathogenesis of rheumatoid arthritis and joint damage. Arthritis Res Ther 2014; 16(2):R99. 
17. Zhukov O, Rivera J, Charles MR, Joanna MP, Stanley JN. Serum 14-3-3n protein supplements traditional rheumatoid arthritis biomarkers. Arthritis Rheum 2014; 66(11):407.

18. Cooper NJ. Economic burden of rheumatoid arthritis: a systematic review. Rheumatology (Oxford) 2000; 39(1):28-33.

19. Finckh $\mathrm{A}$, Liang $\mathrm{MH}$, Van Herckenrode $\mathrm{CM}$, de Pablo P. Long-term impact of early treatment on radiographic progression in rheumatoid arthritis: A meta-analysis. Arthritis Rheum 2006; 55(6):864-72.

20. Puolakka K, Kautiainen H, Pohjolainen T, Virta L. No increased mortality in incident cases of rheumatoid arthritis during the new millennium. Ann Rheum Dis 2010; 69(11):2057-8. 